\title{
Improving outcomes with pharmacotherapy to treat acute esophageal variceal bleeding
}

\author{
C. E. Eapen ${ }^{1} \cdot$ Subramani Kandasamy ${ }^{2}$
}

Published online: 29 August 2018

(C) Indian Society of Gastroenterology 2018

Borrowing from geo-political terminology, the portal circulation between the bowel and liver can be considered as a "landlocked" country. The portal venous circulation starts from venules draining the capillary bed in the intestines, spleen, and pancreas and ends in portal vein branches/venules draining into sinusoids in the liver. This anatomic system peculiarity renders access via the endovascular route to this land-locked countryi.e. the portal circulation - a challenge. One way to obtain entry to this difficult-to-access country is to bore a tunnel through a boundary wall—as done by creating a transjugular intrahepatic portosystemic shunt (TIPSS).

Escalating tensions within this relatively inaccessible country lead to predictable consequences. Portal hypertension manifests as significant deleterious clinical consequences like esophageal or gastric variceal bleeding, ascites, hepatic encephalopathy, and hepatorenal syndrome (HRS). It is important to accurately measure portal venous pressure to understand the pathophysiology of the complications of portal hypertension as well as to study the effects of treatments to reduce portal pressure in these situations. In contrast to highpressure systemic arterial circulation (normal mean arterial pressure is $65-110 \mathrm{mmHg}$ ), the portal venous system is a low-pressure system (normal portal venous pressure is < $5 \mathrm{mmHg}$ ). Invasive methods to assess pressure in the portal circulation include direct measurements from a catheter placed in the portal vein transhepatically (ultrasound guided) or during surgery and indirect methods like hepatic venous

\section{E. Eapen}

eapen@cmcvellore.ac.in

1 Department of Hepatology, Christian Medical College, Vellore 632 004, India

2 Surgical Intensive Care, Christian Medical College, Vellore 632 004, India pressure gradient measurement or endoscopic transducer probe used to assess esophageal variceal pressure. Hepatic venous pressure gradient is considered as the gold standard to measure portal pressures and if this gradient is $\geq 10 \mathrm{mmHg}$, this indicates clinically significant portal hypertension [1]. Non-invasive methodsclinical, biochemical, and physical (elastography) methods-permit periodic assessment of the degree of portal hypertension in patients with chronic liver disease [2].

One strategy to reduce portal pressure in patients with cirrhosis and portal hypertension is to use drugs ("ambassadors") to access the "land-locked" portal circulation — which binds to specific receptors ("embassies") and influence portal pressure. We now have a variety of vasoactive drugs to reduce portal pressure acutely like terlipressin, vasopressin, somatostatin, and octreotide [3]. Of these drugs, terlipressin (acts on vasopressin [V1 a] receptors in the splanchnic vascular smooth muscle [4]) is the clear winner as a portal pressure-reducing drug.

Terlipressin-induced splanchnic vasoconstriction is used to treat HRS (by lowering portal pressure and increasing renal blood flow, is most effective to treat type 1 HRS) and to control acute variceal bleeding (by lowering portal pressure). Pharmacotherapy in combination with endotherapy (band ligation of varices) is the current standard of care to treat acute esophageal variceal bleeding. However, terlipressin can cause life-threatening cardiovascular and perfusion-related complications.

In most of the studies showing benefit of terlipressin in HRS and in acute variceal bleed, the patients received intermittent intravenous boluses of terlipressin. However, portal pressurelowering effects of intravenous terlipressin boluses last only $3-$ $4 \mathrm{~h}$ [5]. Hence, recent studies have explored using continuous infusion of terlipressin and have shown this to be superior to intermittent bolus administration in patients with HRS [6] and undergoing TIPSS insertion [7] in lowering portal pressure stably 
as well as having lesser adverse effects. The safety profile of continuous terlipressin infusion may be related to the lower total dose needed compared to the bolus therapy. Studies are now needed to see if these benefits of continuous infusion over intermittent boluses of terlipressin translate into better clinical outcomes in patients with acute variceal bleed.

In this context, the study by Jha et al. published in this issue of the Journal is useful. In this randomized control trial of terlipressin (given for 5 days) and endoscopic variceal ligation in 86 patients with acute variceal bleed, mostly due to cirrhosis, continuous infusion was superior to intermittent boluses of terlipressin in preventing treatment failure [8]. Treatment failure was defined as failure to achieve control of variceal bleed (by Baveno VI criteria). However, there was no difference in 6-week mortality in both the treatment arms. The incidence of adverse effects of terlipressin was equal in both the treatment arms, which may be related to similar daily total dosing in both the arms during the study period. As mentioned by the authors, the lack of hepatic venous pressure gradient measurement is a limitation of this study. It is unclear how many boluses of terlipressin were administered prior to endotherapy in this study. Further studies are needed to validate these findings.

The indications for terlipressin in patients with cirrhosis continue to expand with its ambulatory administration in patients with terlipressin-responsive HRS while awaiting for liver transplantation, and its use in the treatment of patients with refractory ascites [9].

\section{Compliance with ethical standards}

Conflict of interest CEE, and SK declare that they have no conflict of interest.
Disclaimer The authors are solely responsible for the data and the content of the paper. In no way, the Honorary Editor-in-Chief, Editorial Board Members, or the printer/publishers are responsible for the results/ findings and content of this article.

\section{References}

1. Abraldes JG, Sarlieve P, Tandon P. Measurement of portal pressure. Clin Liver Dis. 2014;18:779-92.

2. de Oliveira AC. Noninvasive assessment of portal hypertension and detection of esophageal varices in cirrhosis: state-of-the-art. Eur J Gastroenterol Hepatol. 2017;29:531-4.

3. Satapathy SK, Sanyal AJ. Nonendoscopic management strategies for acute esophagogastric variceal bleeding. Gastroenterol Clin N Am. 2014;43:819-33.

4. Møller S, Hansen EF, Becker U, Brinch K, Henriksen JH, Bendtsen F. Central and systemic haemodynamic effects of terlipressin in portal hypertensive patients. Liver. 2000;20:51-9.

5. Escorsell A, Bandi JC, Moitinho E, et al. Time profile of the haemodynamic effects of terlipressin in portal hypertension. J Hepatol. 1997;26:621-7.

6. Cavallin M, Piano S, Romano A, et al. Terlipressin given by continuous intravenous infusion versus intravenous boluses in the treatment of hepatorenal syndrome: a randomized controlled study. Hepatology. 2016;63:983-92.

7. Ding C, Wu X, Fan X, He C, Li J. Hemodynamic effects of continuous versus bolus infusion of terlipressin for portal hypertension: a randomized comparison. J Gastroenterol Hepatol. 2013;28:1242-6.

8. Jha SK, Mishra M, Jha A, Dayal VM. Comparison of continuous infusion versus intermittent infusion of terlipressin for the control of acute variceal bleeding in patients with portal hypertension: an openlabel randomized controlled trials. Indian J Gastroenterol. 2018:37. doi.org/10.1007/s12664-018-0871-8

9. Papaluca T, Gow P. Terlipressin: current and emerging indications in chronic liver disease. J Gastroenterol Hepatol. 2018;33:591-8. 